\title{
Ordering in nematic liquid crystals from NMR cross-polarization studies
}

\author{
K V RAMANATHAN ${ }^{*, *}$ and NEERAJ SINHA ${ }^{\dagger}$ \\ ${ }^{*}$ Sophisticated Instruments Facility; ${ }^{\dagger}$ Department of Physics, Indian Institute of Science, \\ Bangalore 560 012, India \\ †Corresponding author \\ Email: kvr@sif.iisc.ernet.in
}

\begin{abstract}
The measurement of dipolar couplings between nuclei is a convenient way of obtatining directly liquid crystalline ordering through NMR since the coupling is dependent on the average orientation of the dipolar vector in the magnetic field which also aligns the liquid crystal. However, measurement of the dipolar coupling between a pair of selected nuclei is beset with problems that require special solutions. In this article the use of cross polarization for measuring dipolar couplings in liquid crystals is illustrated. Transient oscillations observed during cross polarization provide the dipolar couplings between essentially isolated nearest neighbour spins which can be extracted for several sites simultaneously by employing two-dimensional NMR techniques. The use of the method for obtaining heteronuclear dipolar couplings and hence the order parameters of liquid crystals is presented. Several modifications to the basic experiment are considered and their utility illustrated. A method for obtaining proton-proton dipolar couplings, by utilizing cross polarization from the dipolar reservoir, is also presented.
\end{abstract}

Keywords. NMR; cross polarization; liquid crystals; dipolar couplings; ordering; order parameter.

\section{Introduction}

NMR spectroscopy has long been recognized as a valuable tool for the study of liquid crystalline order and dynamics [1,2]. There are a variety of NMR techniques, which provide these informations. Proton-proton dipolar couplings have been one of the major sources of information for the study of small molecules up to about ten spins oriented in liquid crystalline media [3]. However, for larger molecules and for the liquid crystals themselves the method has been of very limited use. On the other hand, measurements of heteronuclear dipolar couplings pose a lesser problem in terms of linewidth and resolution, and therefore offer an attractive means of studying liquid crystalline ordering. One of the first demonstrations of this approach has been made with the study of ${ }^{2} \mathrm{H}-{ }^{13} \mathrm{C}$ dipolar couplings in $N$-(4-methoxybenzylidene)-4- $n$-butylaniline (MBBA) deuterated at a specific site [4]. Similar studies have been performed on systems containing ${ }^{19} \mathrm{~F}$ and using ${ }^{19} \mathrm{~F}-{ }^{13} \mathrm{C}$ dipolar couplings $[5,6]$. However, a more general and attractive approach would be to utilize ${ }^{1} \mathrm{H}-{ }^{13} \mathrm{C}$ dipolar couplings. The use of the 2D separated local field (SLF) procedure 
for this purpose was first demonstrated for the case of MBBA by Hohener et al [4]. The ${ }^{13} \mathrm{C}$ chemical shifts and the corresponding ${ }^{13} \mathrm{C}-{ }^{1} \mathrm{H}$ dipolar splittings have been displayed along the $F_{2}$ and $F_{1}$ axes. However, in this method, the presence of the homonuclear dipolar couplings tend to make the lines broad along the $F_{1}$ axis. The problem of eliminating the proton-proton dipolar couplings has been addressed by employing the variable angle sample spinning (VASS) technique [7]. Due to the off-magic angle spinning, the proton dipolar coupling is significantly reduced and further use of the multiple pulse decoupling during the $t_{1}$ period enables near elimination of proton dipolar couplings. The ${ }^{13} \mathrm{C}-{ }^{1} \mathrm{H}$ dipolar splittings observed along $F_{1}$ are to be suitably scaled to include the effect of VASS and the multiple pulse decoupling, to obtain the actual dipolar couplings. This method has been used for the study of a large number of liquid crystalline systems [8]. Modification to this technique has also been suggested which enables short range and long range dipolar couplings to be measured with better resolution [9-15].

Another approach that has been proposed and implemented is the use of a variant of the SLF 2D experiment that uses the transient oscillations observed during cross polarization [16,17] for estimating dipolar couplings [18]. Modifications to the standard crosspolarization pulse scheme such as the use of homonuclear decoupling during cross polarization (CP) and the use of polarization inversion have also been suggested [19,20]. These are described in more detail in the following sections. A method of indirectly monitoring proton dipolar couplings using the carbon chemical shift dispersion has also been suggested which is also described. The various pulse schemes that have been utilized are shown in figure 1.

\section{Dipolar oscillations in cross polarization}

Observation of dipolar oscillations during CP has been made by Muller et al [16] in a single crystal of ferrocene. In liquid crystals such as MBBA (figure 2), ${ }^{13} \mathrm{C}$ spectra containing sharp well-resolved lines can be obtained using the CP sequence (figure 1a).

The intensity of the carbon lines show an oscillatory build-up when recorded as a function of the contact time $t$ [18]. These oscillations are observed when the dipolar couplings of a carbon to its nearest neighbour protons are much stronger than the coupling of this spin system to the rest of the protons. The frequency $f$ of the oscillations is given by

$$
M_{S}=M_{S 0}(1-\cos (f t)) .
$$

The value of $f$ is given in terms of $D$, the $\mathrm{C}-\mathrm{H}$ dipolar coupling. It also depends on the number of equivalent protons coupled to a carbon.

Thus for an $I S$ spin system, such as a C-H group, $f=D$ and for the $I_{2} S$ spin system such as the $\mathrm{CH}_{2}$ group, $f=\sqrt{2} D$. For the methyl group $\left(I_{3} S\right.$ case $)$, three values for $f$ may be expected corresponding to $D, \sqrt{3} D$ and $2 D$. Here the dipolar coupling $D$ expressed in $\mathrm{kHz}$ is defined as

$$
D=\left(-h \gamma_{\mathrm{C}} \gamma_{\mathrm{H}} / 4 \pi^{2} r_{\mathrm{CH}}^{3}\right) S_{\mathrm{CH}},
$$

where $\gamma_{\mathrm{H}}$ and $\gamma_{\mathrm{C}}$ are the gyromagnetic ratios of the protons and carbons respectively, $r_{\mathrm{CH}}$ is the internuclear distance and $S_{\mathrm{CH}}$ is the order parameter along the direction of the $\mathrm{C}-\mathrm{H}$ vector. The value of $\left(h \gamma_{\mathrm{C}} \gamma_{\mathrm{H}} / 4 \pi^{2} r_{\mathrm{CH}}^{3}\right)$ is $22.68 \mathrm{kHz}$ for $r_{\mathrm{CH}}=1.1 \AA$. Equation (1) is further 


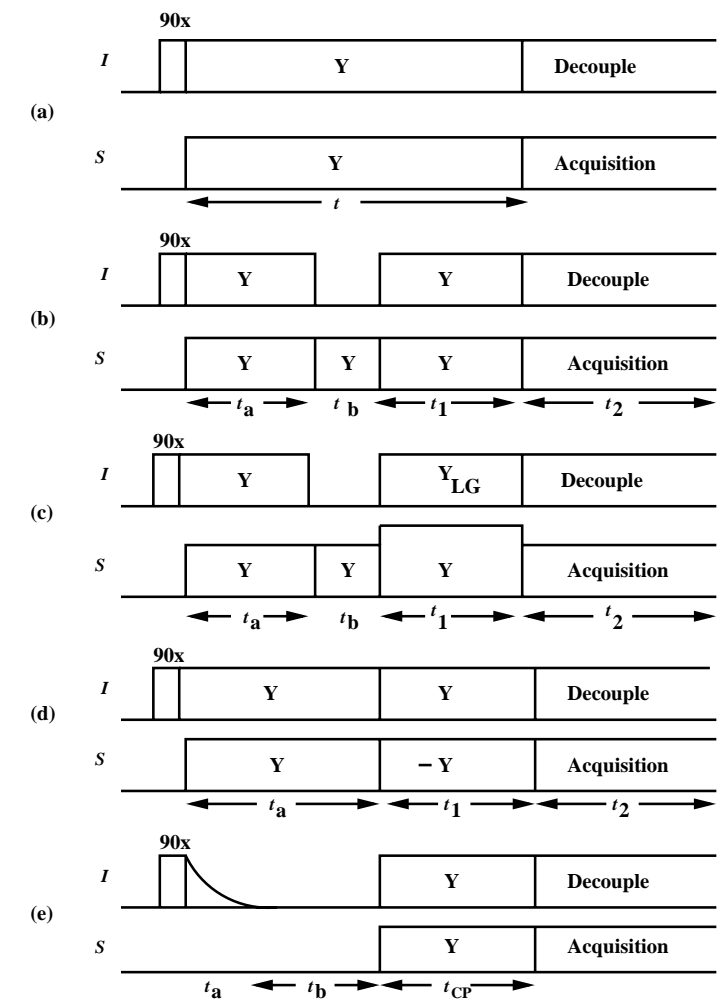

Figure 1. Various cross-polarization pulse schemes employed for obtaining dipolar couplings: (a) Hartmann-Hahn cross polarization, (b) cross depolarization, (c) cross depolarization with Lee-Goldburg decoupling, (d) polarization inversion and (e) cross polarization from the dipolar bath.

modified [16] to take into account spin diffusion between the protons directly coupled to the carbon under consideration and the rest of the protons in the system as

$$
M_{S}=M_{S 0}\left[1-1 / 2 \exp \left(-t / T_{I I}\right)-1 / 2 \exp \left(-3 t / T_{I I}\right) \cos (f t)\right],
$$

where $T_{I I}$ represents the time constant for the spin diffusion process.

Figure 2 shows the proton decoupled ${ }^{13} \mathrm{C}$ spectrum of MBBA at room temperature. Figure 3 presents the variation of the intensities of the $\mathrm{C}_{7}, \mathrm{C}_{\alpha}, \mathrm{C}_{\beta}$, and $\mathrm{C}_{\gamma}$ carbons as a function of the contact time. The oscillation frequency $f$ and hence dipolar coupling $D$ can be obtained by fitting eq. (3) to the experimental data.

The values $3.73,4.84,2.76$, and $2.84 \mathrm{kHz}$ were obtained for $D$ respectively for the $\mathrm{C}_{7}$, $\mathrm{C}_{\alpha}, \mathrm{C}_{\beta}$, and $\mathrm{C}_{\gamma}$ carbons of MBBA. The $\mathrm{C}_{7}$ dipolar coupling enables the order parameter $S$ of the aromatic core to be estimated with the assumption of uniaxial ordering from

$$
D=-S\left(h \gamma_{\mathrm{C}} \gamma_{\mathrm{H}} / 4 \pi^{2} r_{\mathrm{CH}}^{3}\right)\left(3 \cos ^{2} \theta-1\right) / 2,
$$



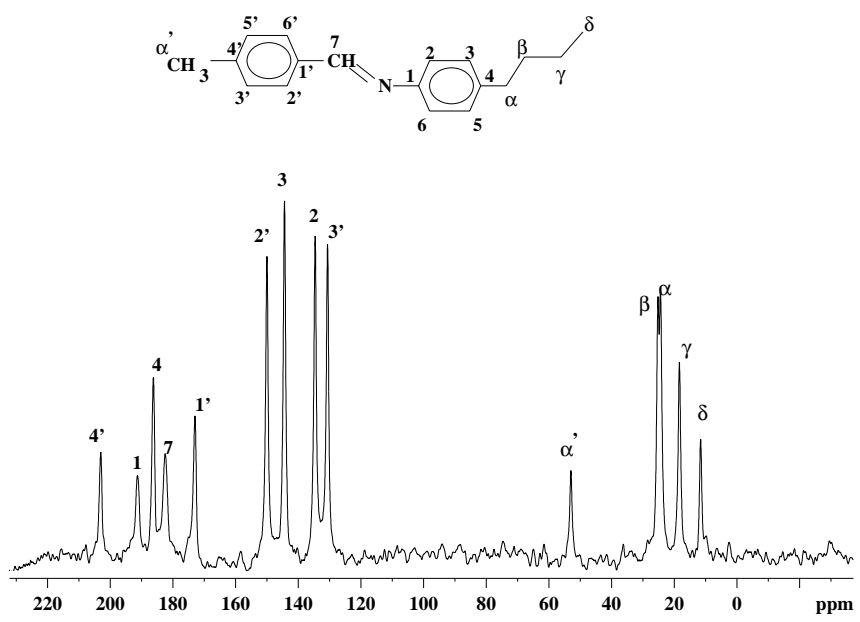

Figure 2. Proton-decoupled ${ }^{13} \mathrm{C}$ spectrum of MBBA recorded on a Bruker DSX-300 NMR spectrometer at $75.47 \mathrm{MHz}$ at room temperature.

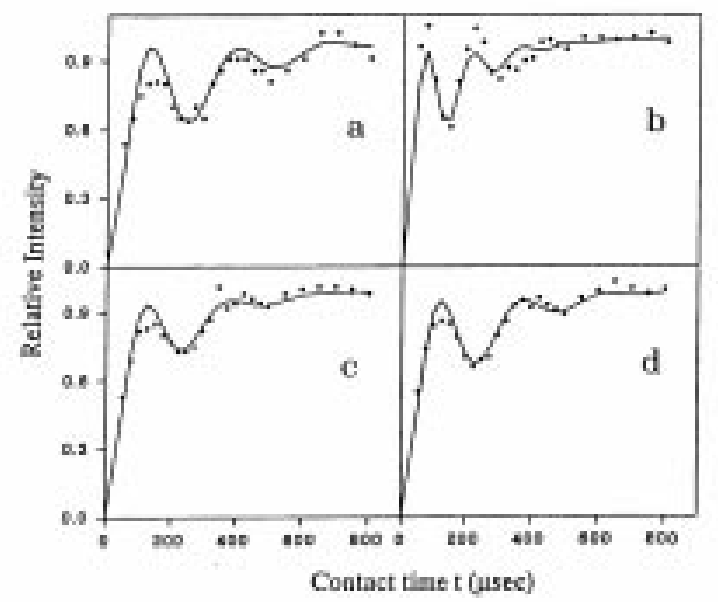

Figure 3. Relative intensities of signals of (a) $C_{7}$, (b) $C_{\alpha}$, (c) $C_{\beta}$, and (d) $C_{\gamma}$ carbons of MBBA plotted against contact time. Points correspond to experimental values and the continuous line corresponds to the fit to the data in eq. (3) with (a) $f=3.73 \mathrm{kHz}$, $T_{I I}=333 \mu \mathrm{s}$, (b) $f=6.84 \mathrm{kHz}, T_{I I}=181 \mu \mathrm{s},(\mathbf{c}) f=3.90 \mathrm{kHz}, T_{I I}=228 \mu \mathrm{s}$, and (d) $f=4.0 \mathrm{kHz}, T_{I I}=273 \mu \mathrm{s}$.

where $\theta$ is the angle between the director and the $\mathrm{C}-\mathrm{H}$ bond. Using a value of $114^{\circ}$ for the $\mathrm{C}-\mathrm{C}-\mathrm{H}$ bond angle and assuming a tilt of the director by $3.5^{\circ}$ from the para-axis of the adjacent phenyl ring, a value of $S=0.52$ was obtained from these measurements, which agrees well with values reported by using other methods. The magnitude of the order parameter along the aliphatic chain averaged over several conformations can also be calculated from eq. (2) using the experimentally obtained values of dipolar couplings. The 


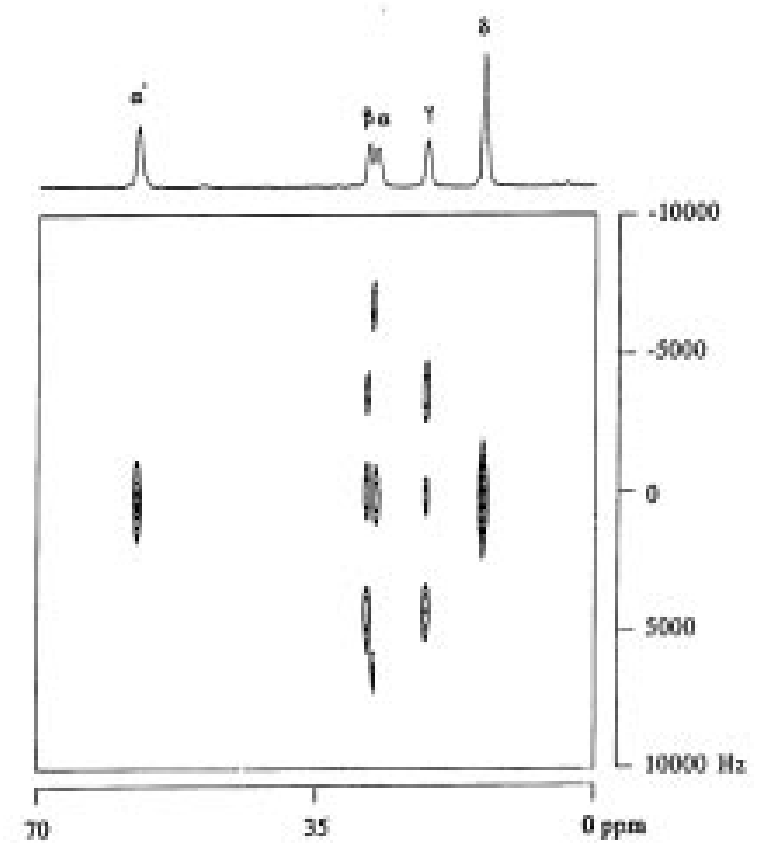

Figure 4. Two-dimensional spectrum of the aliphatic carbons of MBBA obtained using the pulse sequence shown in figure $1 \mathrm{~b}$ on a Bruker DSX-300 NMR spectrometer at $75.47 \mathrm{MHz}$ at room temperature. The horizontal axis corresponds to ${ }^{13} \mathrm{C}$ chemical shifts and the vertical axis to the dipolar oscillation frequencies. The projection along the horizontal axis of the two-dimensional spectrum is also shown.

values $0.21,0.12$, and 0.13 obtained for the carbon sites $\alpha, \beta$, and $\gamma$ are in agreement with the variation of order parameter generally observed in liquid crystalline systems.

A straightforward way of obtaining the oscillation frequencies is to use the 2D approach where the CP contact time $(t)$ is incremented in regular steps between successive experiments. A two-dimensional data set is collected with proton decoupling during $t_{2}$. A two-dimensional Fourier transform then gives ${ }^{13} \mathrm{C}$ chemical shifts along the $F_{2}$ axis and the oscillation frequencies along $F_{1}$. A cross-depolarization experiment (figure $1 \mathrm{~b}$ ), rather than a cross-polarization experiment provides better results, the axial peaks which arise from the non-oscillatory terms of eq. (3) are less intense in the former case (vide infra). The $S$ spin magnetization during the $t_{1}$ period is given by

$$
M_{S}=M_{S 0}\left[1 / 2 \exp \left(-t_{1} / T_{I I}\right)+1 / 2 \exp \left(-3 t_{1} / T_{I I}\right) \cos \left(f t_{1}\right)\right] .
$$

The peaks along the $F_{1}$ axis provide the oscillation frequencies $f$ from which the dipolar couplings can be estimated using the prescription provided earlier. Results of such an experiment carried out with $t_{a}=1 \mathrm{~ms}$ and $t_{b}=200 \mu \mathrm{s}$ are shown in figure 4 for the aliphatic carbons of MBBA. There are cross peaks occurring at $6.8,3.8$, and $4.0 \mathrm{kHz}$ corresponding to the $\mathrm{C}_{\alpha}, \mathrm{C}_{\beta}, \mathrm{C}_{\gamma}$ carbons, which provide the corresponding $\mathrm{C}-\mathrm{H}$ dipolar couplings as 4.8 , 2.7 , and $2.8 \mathrm{kHz}$. 


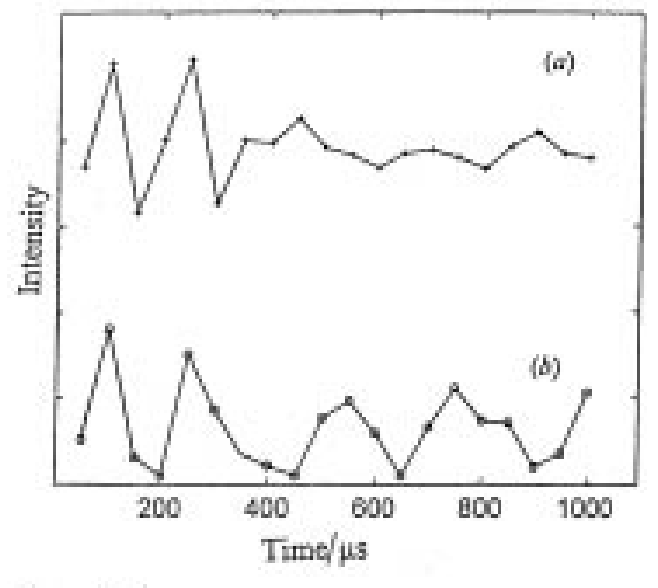

Figure 5. Intensities of the benzylidene carbon of EBBA, as a function of $t_{1}$ obtained after Fourier transformation along $t_{2}$ of the two-dimensional data-set obtained using the pulse scheme shown in figure $1 \mathrm{c}$; (a) with on-resonance proton r.f. during $t_{1}$ and (b) with LG decoupling during $t_{1}$.

\section{Improved schemes}

\subsection{Inclusion of homonuclear decoupling during $C P$}

The dipolar oscillations during $\mathrm{CP}$ are highly damped due to the couplings among the protons. Consequently the peaks in the two-dimensional spectra are broad and in several instances hard to observe. Wu et al [21] have shown that the use of Lee-Goldburg (LG) decoupling [22] during cross polarization results in the removal of homonuclear dipolar couplings leading to a reduction of the linewidth along the dipolar axis. The use of the method has been made for the case of the liquid crystal $N$-(4-ethoxybenzylidene)-4-n-butylaniline (EBBA) [19]. The pulse sequence used for the 2D experiment is shown in figure 1c. The $t_{1}$ period corresponds to transfer of polarization from carbons to protons. During this period the proton offset is changed to satisfy the Lee-Goldburg condition and carbon power level is adjusted to satisfy the Hartmann-Hahn condition so that magnetization exchange takes place under homonuclear proton spin decoupling. The effect of LG decoupling resulting in the lengthening of the dipolar oscillation is shown for the case of the benzylidene carbon of EBBA in figure 5 where results of two experiments, viz., one in which the proton r.f. is applied on-resonance corresponding to normal depolarization experiment and the other in which LG decoupling is employed, are compared.

The homonuclear decoupling during the $t_{1}$ period results in the scaling of the heteronuclear couplings, the theoretical scaling factor being $\sin \left(\theta_{\mathrm{m}}\right)=0.82$, where $\theta_{\mathrm{m}}$ is the magic angle. The two-dimensional spectrum of EBBA was obtained at several temperatures using the method and $\mathrm{C}-\mathrm{H}$ dipolar couplings at several sites were obtained. From the dipolar couplings of the benzylidene carbon, the order parameter of the aromatic core was obtained at these temperatures. Oscillation frequencies were obtained for the aromatic carbons also. From the values of order parameter of the core at different temperatures, the proton-carbon 
dipolar couplings of the aromatic core can be calculated, assuming a hexagonal geometry of the phenyl ring and fast flip motion about the para axis. In this case, the one bond $\mathrm{C}-\mathrm{H}$ dipolar coupling $D_{\mathrm{CH}}^{1}$ is nearly the same as the coupling of this carbon to its orthoproton $D_{\mathrm{CH}}^{2}$ due to the effect of ordering being different along different directions. In such cases, where the carbon is coupled to more than one proton, the evolution of magnetization is expected to proceed in a locked mode [17] and the oscillation frequency is given by $\sqrt{\left[\left(D_{\mathrm{CH}}^{1}\right)^{2}+\left(D_{\mathrm{CH}}^{2}\right)^{2}\right]}$. The values thus calculated and the experimental oscillation frequencies are observed to show a good correlation [19].

\subsection{Polarization inversion}

As pointed out earlier, eq. (3) governs the frequencies and intensities of the peaks along the dipolar axis in the $2 \mathrm{D}$ experiments. The oscillatory cosine term gives rise to the cross peaks containing dipolar coupling information. There is also the non-oscillatory part which gives rise to the zero frequency peaks, which may overlap with cross peaks close to the center, causing difficulties in measuring small dipolar couplings. For attenuating the zero frequency peaks, polarization inversion instead of cross polarization has been utilized [20,21]. The polarization transfer process for a two-spin $I-S$ system can be modelled as a coherent process in mutually commuting zero-quantum and double-quantum manifolds [23]. Under the assumption of high r.f. fields, the dipolar coupling causes an oscillatory evolution of the density matrix in the zero quantum frame, while in the double quantum frame the density matrix remains constant. The former gives rise to the cross peaks and the latter to the axial peak. In polarization inversion (figure 1d) there is an initial polarization transfer from $I$ spins to $S$ spins during $t_{a}$. At the end of this period the two spin system can be taken to be completely polarized due to contact with the proton bath. Thus the initial density matrix $I_{Z}=I_{Z}^{\Sigma}+I_{Z}^{\Delta}$ tends to become $I_{Z}+S_{Z}=2 I_{Z}^{\Sigma}$, where $I_{Z}^{\Sigma}=1 / 2\left(I_{Z}+S_{Z}\right)$ and $I_{Z}^{\Delta}=1 / 2\left(I_{Z}-S_{Z}\right)$ are the density operators in the double- and zero-quantum frames respectively. Inversion of the r.f. field for the $S$ spin corresponds at this point to a change of sign of the $S$ spin Hamiltonian or equivalently the density matrix can be thought of as equal to $I_{Z}-S_{Z}=2 I_{Z}^{\Delta}$. As a result, the initial evolution of magnetization during polarization inversion takes place only in the zero-quantum sub-space. Therefore, the dipolar cross peaks are much more intense than the axial peaks enabling even small dipolar couplings to be observed. At larger contact times, the double-quantum evolution does come into play [20] and the equation governing this process is given by

$$
M_{S}=M_{S 0}\left[1-\exp \left(-t_{1} / T_{I I}\right)-\exp \left(-3 t_{1} / T_{I I}\right) \cos \left(f t_{1}\right)\right] .
$$

Figure 6 shows the contribution to the $S$ spin intensity from the zero-quantum and the double-quantum evolution as well as the combined effect for both cross polarization and polarization inversion experiments. It is observed that polarization inversion leads to a doubling of the amplitude of the oscillatory part and also to a reduction in the initial value of the non-oscillatory component. In a 2D experiment, this would lead to a doubling of the cross-peak intensity and a significant reduction of the axial peak intensity.

A simulation of the relative intensities of the cross peaks in comparison to the axial peaks for three experiments, namely (i) cross polarization, (ii) cross depolarization and (iii) polarization inversion are shown in figure 7, using respectively eqs (3), (5), and (6). 

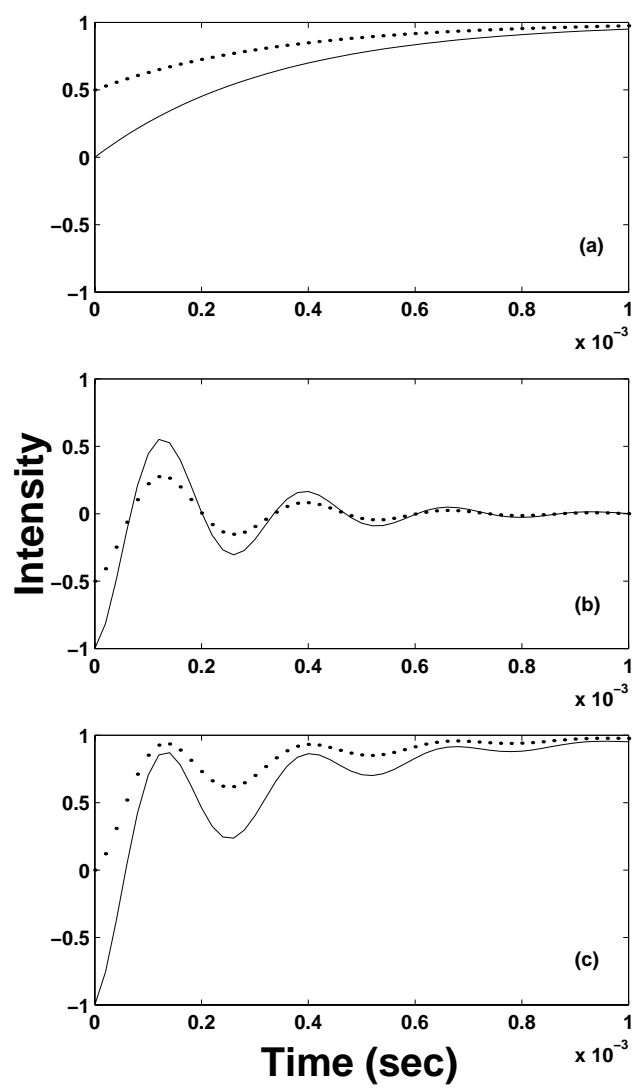

Figure 6. $S$ spin magnetization evolution due to (a) double-quantum process, (b) zero-quantum process, and (c) combined effect of the results shown in (a) and (b) during polarization (....) and polarization inversion (-). The plots correspond to values of $f$ and $T_{I I}$ being $3.73 \mathrm{kHz}$ and $333 \mu \mathrm{s}$.

A dipolar coupling of $9.73 \mathrm{kHz}$ and two different values of $T_{I I}$ equal to $330 \mu \mathrm{s}$ and $1 \mathrm{~ms}$ were considered. It was observed that polarization inversion provides the highest crosspeak intensity for both the values of $T_{I I}$ considered, while cross depolarization provides higher relative cross-peak intensity in comparison to cross polarization.

Experimental demonstration of the usefulness of polarization inversion has been carried out for the case of MBBA [20]. The 2D spectra obtained using cross polarization and polarization inversion are displayed in figure 8 . In the spectrum obtained using polarization inversion shown in figure 8a, most of the carbon-proton dipolar couplings are observed to be resolved. For several carbons the cross peaks are much more intense than the axial peaks such that they are not seen in the plot shown in figure 8a. Some typical cross-sections are shown in figure 9. It is interesting to note that not only short range dipolar couplings of carbons with attached protons could be obtained, but also those of quaternary carbons coupled to remote protons are resolved. This will then lead to useful information on the order parameters of the system studied. 

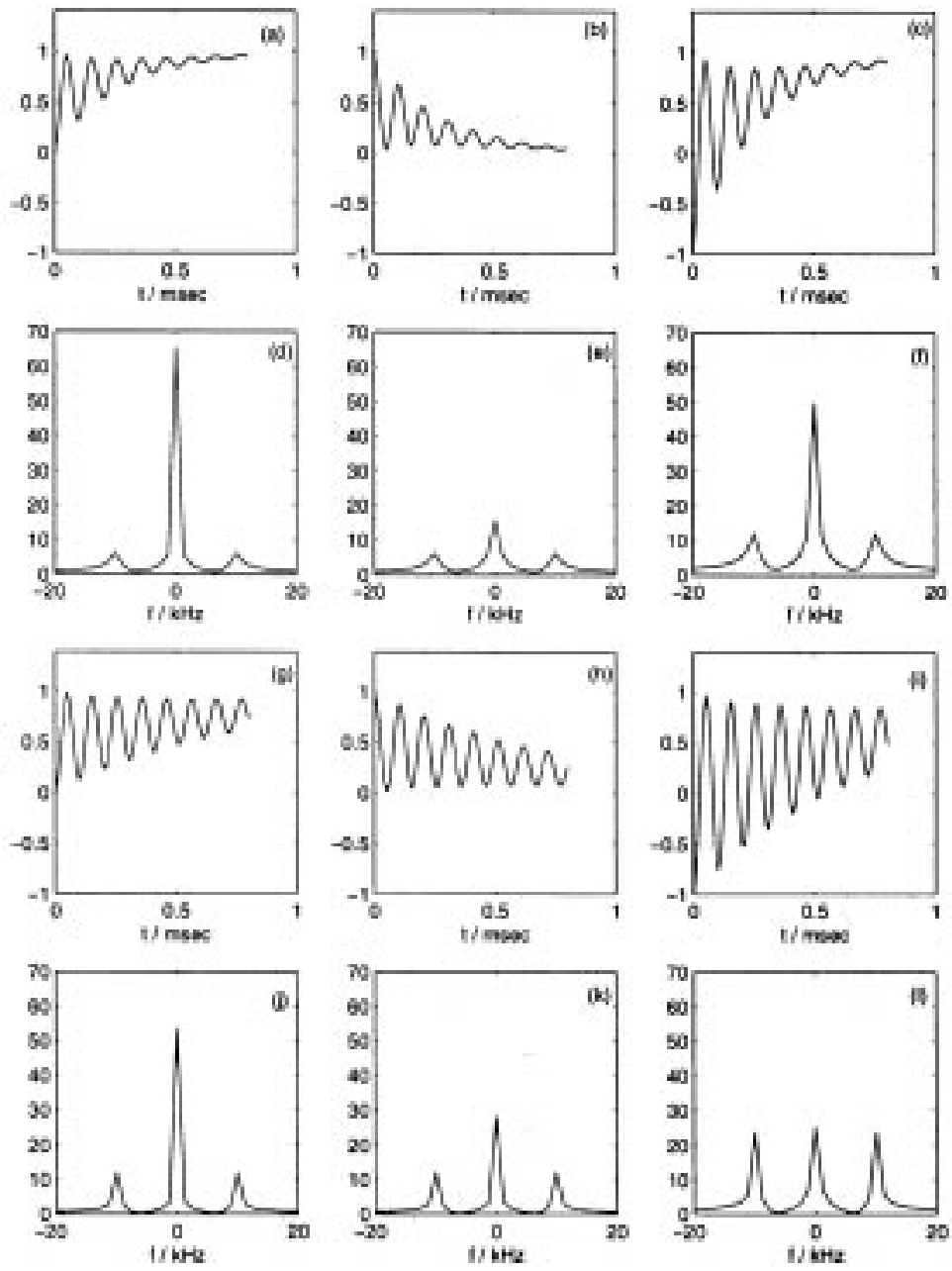

Figure 7. Comparison of cross-peak intensity vs. axial peak intensity for three different cross-polarization experiments. The time domain signals have been obtained using eqs (3), (5), and (6). The corresponding Fourier transforms are shown one below the other. The first, second and third columns correspond to polarization, depolarization, and polarization inversion experiments. The dipolar coupling used is $9.73 \mathrm{kHz}$ for all the cases, $T_{I I}$ is $330 \mu$ s for the top two rows and $1 \mathrm{~ms}$ for the two bottom rows.

\section{Cross polarization from the dipolar reservoir under mis-matched Hartmann-Hahn condition}

In the studies reported so far, polarization transfer has been considered between the Zeeman reservoirs of $I$ and $S$ spins under Hartmann-Hahn match. For a mismatch of the Hartmann-Hahn condition, an absorptive Lorentzian behaviour for the $S$ spin 

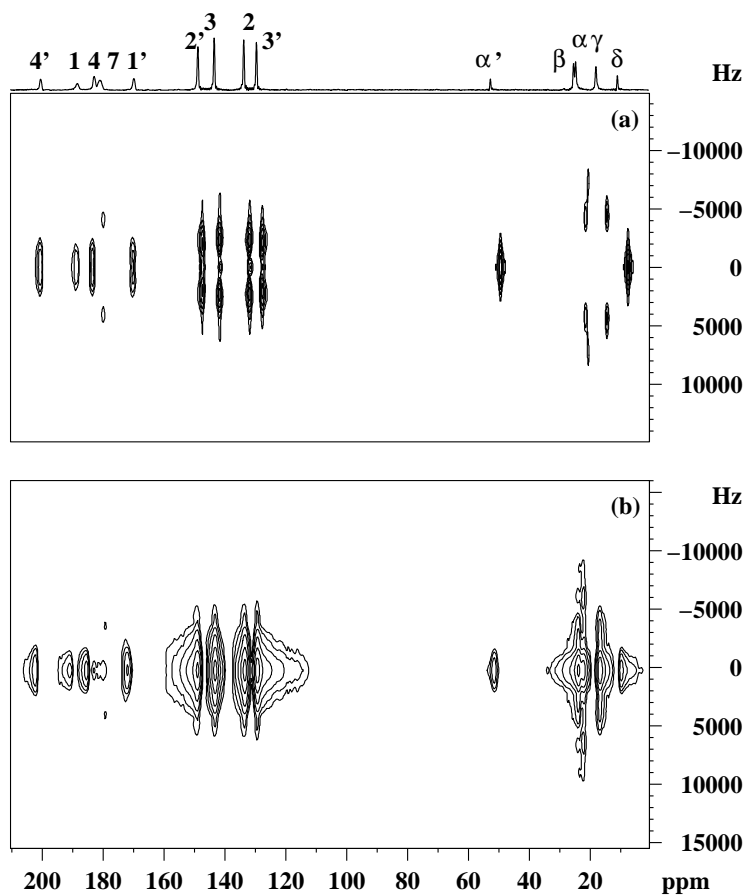

Figure 8. ${ }^{13}$ C SLF-2D NMR spectra of MBBA in its nematic phase obtained with (a) polarization inversion and (b) standard cross polarization pulse schemes on a Bruker DSX-300 NMR spectrometer at room temperature.

intensity has been predicted [23] with the width of the Lorentzian being related to the homonuclear dipolar coupling of the abundant spins. A similar study in which the initial magnetization is in the dipolar bath rather than in the $I$ spin Zeeman bath has also been reported [24] and on the basis of the quasi-equilibrium theory a dispersive Lorentzian behaviour for the $S$ spin intensity has been predicted. The above polarization transfer process has been considered in detail by carrying out experimental measurements for a range of mismatch conditions by utilizing the pulse scheme shown in figure 1e [25]. Here, an ADRF pulse sequence on the $I$ spins creates a dipolar order from the $I$ spin Zeeman order during the period $t_{a}$. During $t_{\mathrm{CP}}$, a Hartmann-Hahn cross-polarization pulse sequence is used, which results in the transfer of polarization from the dipolar bath to the $S$ spin Zeeman bath. The transfer process has the characteristics that $S$ spin intensity is zero for $\omega_{1 S}=\omega_{1 I}$, positive for $\omega_{1 S}>\omega_{1 I}$ and negative for $\omega_{1 S}<\omega_{1 I}$, where $\omega_{1 S}$ and $\omega_{1 I}$ are the strengths of the spin-lock r.f. on the $S$ and $I$ spins respectively during the $\mathrm{CP}$ process. The $S$ spin intensity plotted as a function of $\Delta \omega=\omega_{1 S}-\omega_{1 I}$ is given by

$$
M_{S}=\frac{\beta \lambda^{2} \Delta \omega}{\Delta \omega^{2}+\lambda^{2}},
$$

where $\beta$ is the initial inverse spin temperature of the dipolar reservoir and $\lambda^{2}$ is related to the $I$ spin second moment. The experimental results for the $\alpha$ carbon of EBBA in its oriented phase obtained by observing the ${ }^{13} \mathrm{C}$ resonance and by using the pulse sequence 


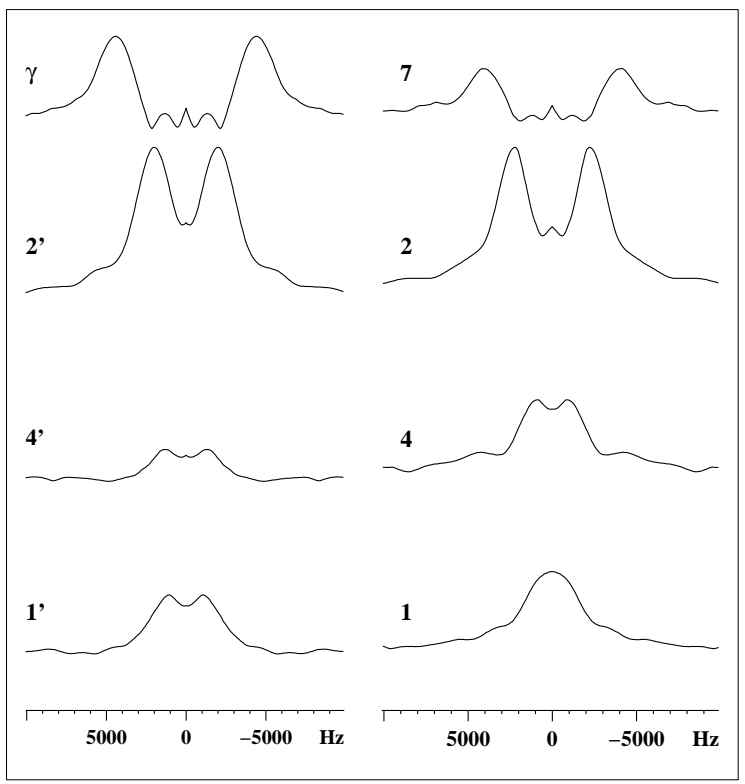

Figure 9. Plots of cross-sections along the $F_{1}$ dimension giving the dipolar oscillation frequencies for $\gamma, 7,2^{\prime}, 2,4^{\prime}, 4,1^{\prime}$, and 1 carbons of MBBA obtained from the 2D plot shown in figure $8 \mathrm{a}$.

of figure 1e are shown in figure 10. The points correspond to the intensity of the carbon line as a function of $\omega_{1 S}$ for a fixed value of $\omega_{1 I}$.

It is indeed observed that the plot shows an overall dispersive Lorentzian behaviour. However, the fit to the data in eq. (7) showed some deviations. A better fit to the experimental data could be obtained by including an additional adsorptive Lorentzian of the same width which could arise due to transfer from remnant Zeeman magnetization of the $I$ spins at the end of the ADRF period, cross polarizing directly to the $S$ spins [25]. The continuous curve shown in the figure corresponds to the fit obtained using the above procedure. A value $14.2 \mathrm{kHz}^{2}$ for $\lambda^{2}$ has been obtained for the $\alpha$ carbon of EBBA. Other methylene carbons along the chain show a variation of $\lambda^{2}$ that is expected on the basis of the variation of the local order parameters of the system. This method like the WISE technique [26], provides a means of indirectly monitoring proton dipolar couplings using the heteronuclear chemical shift dispersion.

\section{Applications}

The cross-polarization techniques mentioned above have been utilized to obtain dipolar couplings and order parameters in several novel liquid crystalline systems such as

(i) Aromatic rings containing four rings in the main core, a lateral hexyloxy chain, and a lateral aromatic branch with the aromatic ring itself being modified by different substituents at meta or para position [27]. 


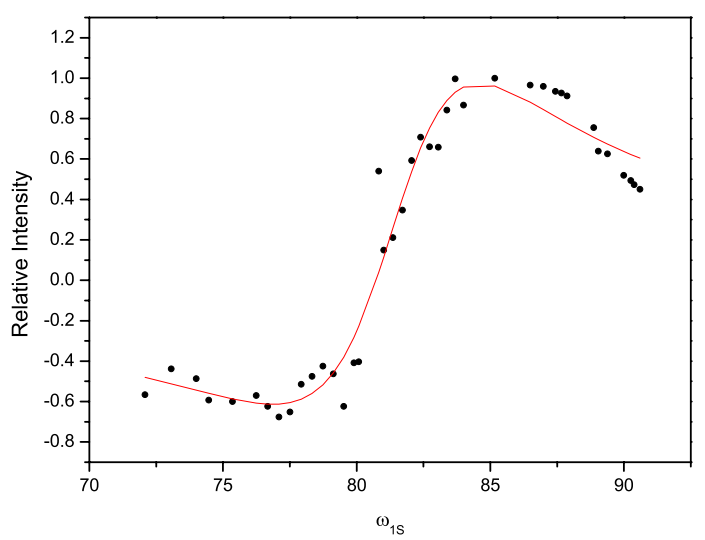

Figure 10. Variation of cross-polarized signal intensity from the dipolar bath of protons to the $\alpha$-carbons in the liquid crystal EBBA for different r.f. powers $\left(\omega_{1 S}\right)$ on ${ }^{13} \mathrm{C}$. The experimental points have been obtained using the pulse sequence of figure 1e, with $t_{a}=t_{b}=1 \mathrm{~ms}$ and $t_{\mathrm{CP}}=200 \mu \mathrm{s}$. The spectra were obtained on a Bruker DSX-300 NMR spectrometer at a ${ }^{13} \mathrm{C}$ resonance frequency of $75.43 \mathrm{MHz}$. The proton r.f. power $\left(\omega_{1 I}\right)$ was kept constant at $83 \mathrm{kHz}$. The continuous curve corresponds to a fit to the experimental data to a mixture of dispersive and abrosptive Lorentzian functions and yields a value of $\lambda^{2}=14.2 \mathrm{kHz}^{2}$ for the $\alpha$ carbon.

(ii) Systems containing four rings in the main core, one terminal, and two nearby lateral chains on each of the outer aromatic rings [28].

(iii) Molecules containing the 2-phenylindazole core - the first bonds of the two terminal chains on either side are not along the same axis due to the presence of the five membered ring in the core [29].

As an illustration of the use of the $\mathrm{C}-\mathrm{H}$ bond order parameter obtained from utilizing the cross-polarization technique, systems containing three aromatic rings with a lateral crown-ether fragment and with oxyethylene (OE) units replacing the terminal alkoxy chains is presented [30] here. The replacement of the alkoxy chains by chains containing the oxyethylene units decreases the melting and clearing temperature so as to obtain nematic compounds near room temperature. The symmetric mesogen containing one $\mathrm{OE}$ unit in the terminal chain, referred to as CINPOE1Bu (figure 11) has been studied. The ${ }^{13} \mathrm{C}$ chemical shifts in the isotropic phase and in the nematic phase at different temperatures have been monitored. The SLF 2D spectrum of the compound in its nematic phase at $349 \mathrm{~K}$ obtained using the pulse sequence of figure $1 \mathrm{~d}$ is shown in figure 11. From the peaks in dipolar dimension, the magnitude of the dipolar interaction for several carbons have been obtained.

For example, carbon $\mathrm{C}_{a}$ and $\mathrm{C}_{c}$ have the same chemical shifts, but have different dipolar couplings, the larger one being attributed to $\mathrm{C}_{a}$, the first carbon in the terminal chain. In the crown-ether segment, it is noticed that the dipolar couplings decrease from $\mathrm{C}_{\alpha}$ to $\mathrm{C}_{\gamma}$, but increases for $\mathrm{C}_{\delta}$. This indicates that the average angle between the $\mathrm{C}-\mathrm{H}$ bond and the long molecular axis is less than the magic angle for carbons, $\mathrm{C}_{\alpha}$, nearer to the magic angle for $\mathrm{C}_{\beta}$ and $\mathrm{C}_{\gamma}$ and greater than the magic angle for $\mathrm{C}_{\delta}$. This conclusion is supported by molecular modelling studies of the central part of a single molecule. For carbons in 


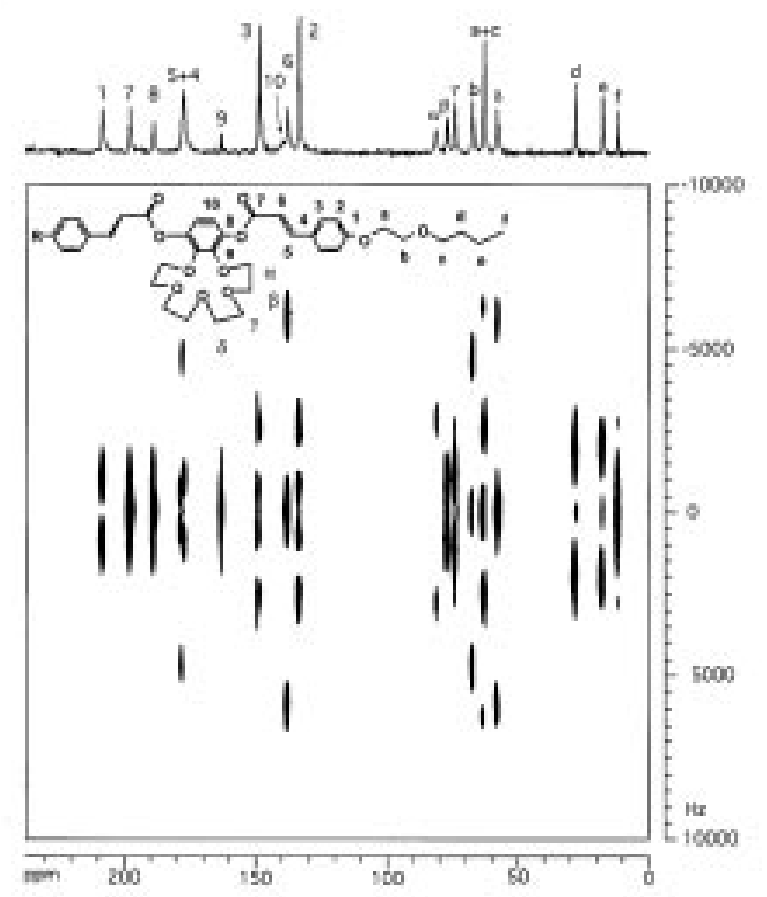

Figure 11. ${ }^{13} \mathrm{C} 2 \mathrm{D}$ spectrum of CINPOE1Bu, a liquid crystal containing a crown-ether and an oxyethylene unit in its nematic phase at $349 \mathrm{~K}$, recorded on a Bruker DSX-300 NMR spectrometer at $75.47 \mathrm{MHz}$.

the polyoxyethylene chain, it is expected that the order parameter decreases monotonically with increasing distance from the core. This is in contrast to the odd-even effect usually observed in a terminal alkyl or alkoxy chain. Such a difference arises due to different probabilities for the conformers of the POE chain [31,32]. In the system under consideration with one OE unit and one butyl fragment, the dipolar couplings decrease monotonically over the OE segment from carbon $a$ to $c$ while for the remaining carbons $(d$ to $f)$, the oddeven effect is present. The variation of the bond order parameter with temperature shows interesting correlation with the corresponding chemical shift of the carbon. A linear correlation between chemical shift and order parameter indicates that the conformation does not change significantly in the temperature range studied. Such a linear correlation has been observed for the carbons of the lateral crown-ether. On the other hand, the non-linear correlation observed for the carbons of the $\mathrm{OE}$ unit indicates that the conformational probabilities change significantly with temperature resulting in different averaged values of the dipolar couplings and chemical shift anisotropies.

\section{Conclusions}

NMR studies using cross polarization have been shown to provide detailed information on dipolar couplings in nematic liquid crystalline systems. The information thus obtained enables estimation of the order parameters of the liquid crystals. Several variants of the 
standard cross-polarization experiments were presented and their utility discussed. Typical applications of the method were presented. In addition to the above studies where heteronuclear dipolar couplings were used, another cross-polarization method that can provide information about the proton-proton dipolar couplings was examined, thus providing another means of obtaining local order.

\section{Acknowledgements}

The authors would like to thank Prof. Anil Kumar for useful suggestions.

\section{References}

[1] J W Emsley, Nuclear magnetic resonance of liquid crystals (Reidel, Dordrecht, 1985)

[2] R Y Dong, Nuclear magnetic resonance of liquid crystals (Springer, New York, 1994)

[3] P Diehl and C L Khetrapal, NMR: Basic principles and progress 1, 1 (1969)

[4] A Hohener, L Muller and R R Ernst, Mol. Phys. 38, 909 (1979)

[5] M L Magnuson, L F Tanner and B M Fung, Liq. Cryst. 16, 857 (1994)

[6] M L Magnuson, B M Fung and M Schadt, Liq. Cryst. 19, 333 (1995)

[7] J Courtieu, J P Bayle and B M Fung, Prog. Nucl. Magn. Reson. Spectrosc. 26, 141 (1994)

[8] B M Fung, Encyclopedia of NMR 4, 2744 (1996)

[9] K Schmidt-Rohr, D Nanz, L Emsley and A Pines, J. Phys. Chem. 98, 6668 (1994)

[10] M Hong, K Schmidt-Rohr and A Pines, J. Am. Chem. Soc. 117, 3310 (1995)

[11] S Caldarelli, M Hong, L Emsley and A Pines, J. Phys. Chem. 100, 18696 (1996)

[12] M Hong, A Pines and S Caldarelli, J. Phys. Chem. 100, 14815 (1996)

[13] S Caldarelli, A Lesage and L Emsley, J. Am. Chem. Soc. 118, 12224 (1996)

[14] B M Fung, K Ermolaev and Y Yu, J. Magn. Reson. 138, 28 (1999)

[15] B M Fung, Prog. Nucl. Magn. Reson. 41, 171 (2002)

[16] L Muller, Anil Kumar, T Baumann and R R Ernst, Phys. Rev. Lett. 32, 1402 (1974)

[17] R K Hester, J L Ackerman, V R Cross and J S Waugh, Phys. Rev. Lett. 34, 993 (1975)

[18] R Pratima and K V Ramanathan, J. Magn. Reson. A118, 7 (1996)

[19] C S Nagaraja and K V Ramanathan, Liq. Cryst. 26, 17 (1999)

[20] Neeraj Sinha and K V Ramanathan, Chem. Phys. Lett. 332, 125 (2000)

[21] C Wu, A Ramamoorthy and S J Opella, J. Magn. Reson. A109, 270 (1994)

[22] M Lee and W I Goldburg, Phys. Rev. A140, 1261 (1965)

[23] M H Levitt, D Suter and R R Ernst, J. Chem. Phys. 84, 4243 (1986)

[24] S Zhang, E O Stejskal, R E Fornes and X Wu, J. Magn. Reson. A104, 177 (1993)

[25] T N Venkatraman, Neeraj Sinha and K V Ramanathan, J. Magn. Reson. 157, 137 (2002)

[26] K Schmidt-Rohr, J Clauss and H W Spiess, Macromolecules 25, 3273 (1992)

[27] P Berdague, J P Bayle, H Fujimori and S Miyajima, New. J. Chem. 1005 (1998)

[28] P Berdague, M Munier, P Judeinstein, J P Bayle, C S Nagaraja and K V Ramanathan, Liq. Cryst. 26, 211 (1999)

[29] P Berdague, P Judeinstein, J P Bayle, C S Nagaraja, Neeraj Sinha and K V Ramanathan, Liq. Cryst. 28, 197 (2001)

[30] Neeraj Sinha, K V Ramanathan, P Berdague, P Judeinstein and J P Bayle, Liq. Cryst. 29, 449 (2002)

[31] V Rayssac, P Judeinstein, J P Bayle, D Kuwahara, H Ogata and S Miyajima, Liq. Cryst. 25, 427 (1998)

[32] E T Samulski and R Y Dong, J. Chem. Phys. 77, 5090 (1982) 